\title{
TITLE:
}

\section{Influence of cross-linking density on volume phase transition of liquid crystalline gels in a nematogenic solvent}

\author{
$\operatorname{AUTHOR}(S)$ :
}

Okuno, Y; Urayama, K; Kohjiya, S

\section{CITATION:}

Okuno, Y ... [et al]. Influence of cross-linking density on volume phase transition of liquid crystalline gels in a nematogenic solvent. JOURNAL OF CHEMICAL PHYSICS 2003, 118(21): 9854-9860

\section{ISSUE DATE:}

2003-06-01

URL:

http://hdl.handle.net/2433/39750

\section{RIGHT:}

Copyright 2003 American Institute of Physics. This article may be downloaded for personal use only. Any other use requires prior permission of the author and the American Institute of Physics. 


\title{
Influence of cross-linking density on volume phase transition of liquid crystalline gels in a nematogenic solvent
}

\author{
Yuko Okuno \\ Institute for Chemical Research, Kyoto University, Uji, Kyoto-fu 611-0011, Japan \\ Kenji Urayama ${ }^{a)}$ \\ Department of Material Chemistry, Graduate School of Engineering, Kyoto University, \\ Kyoto 606-8501, Japan \\ Shinzo Kohjiya \\ Institute for Chemical Research, Kyoto University, Uji, Kyoto-fu 611-0011, Japan
}

(Received 27 December 2002; accepted 3 March 2003)

\begin{abstract}
The effect of cross-linking density $\left(C_{x}\right)$ on volume phase transition of two liquid crystalline (LC) networks comprising dissimilar mesogens in a low molar mass LC has been investigated. Cross-linking density alters the modulus and nematicity of LC network each of which directly influence one of the three governing forces (elastic force, nematic interaction, isotropic mixing interaction) for the equilibrium swelling: An increment in $C_{x}$ increases the network modulus, while it reduces the nematic-isotropic transition temperature of the pure (dry) LC network $\left(T_{\mathrm{NI}}^{N}\right)$. The LC networks in the nematogen are discontinuously transformed from the swollen state into the shrunken state, as a result of a single nematic phase formation inside the gel at $T_{\mathrm{NI}}^{G}$. It has been found that as $C_{x}$ increases, the magnitude of discontinuous volume change at $T_{\mathrm{NI}}^{G}$ decreases; $T_{\mathrm{NI}}^{G}$ shifts to higher temperatures, which is opposite to the $C_{x}$ dependence of $T_{\mathrm{NI}}^{N}$. As an extreme case of high cross-linking density, the LC networks with $C_{x}=10 \mathrm{~mol} \%$ in the nematic solvent exhibits the nematic-isotropic transition but do not undergo appreciable volume transition. The experimental swelling-temperature curves are compared with the prediction of a mean field theory for nematic gel. The theory successfully describes the effect of cross-linking density on the swelling and phase behavior observed. (C) 2003 American Institute of Physics. [DOI: 10.1063/1.1569471]
\end{abstract}

\section{INTRODUCTION}

Some polymer networks swollen in solvents are known to undergo a reversibly discontinuous volume change (volume transition) in response to an infinitesimal environmental change in solvent composition, $p \mathrm{H}$, and temperature. ${ }^{1,2} \mathrm{In}$ our previous studies, ${ }^{3,4}$ we first reported that the liquid crystalline (LC) polymer networks in low molar mass nematic LCs discontinuously changed from the isotropic swollen state to the nematic shrunken state, namely, volume transition accompanying the nematic-isotropic transition of the gels. Of further interest was that upon further cooling, in the temperature ( $T$ ) region $T_{\mathrm{NI}}^{S}<T<T_{\mathrm{NI}}^{G}$, where $T_{\mathrm{NI}}^{S}$ and $T_{\mathrm{NI}}^{G}$ are, respectively, the nematic-isotropic transition temperatures outside and inside the gels, the shrunken nematic gels surrounded by isotropic LC solvents swelled again. These swelling and phase characteristics were universal for various combinations of the nematic networks in the dissimilar nematogens including a nematic network in a similar nematogen. ${ }^{4}$ It was also shown ${ }^{4}$ that a mean field theory for nematic gel $^{5-7}$ well described the equilibrium swelling and phase behavior observed. The swelling of nematic gels is primarily governed by the nematic orders of the LC mol-

\footnotetext{
a) Author to whom correspondence should be addressed. Electronic mail: urayama@rheogate.polym.kyoto-u.ac.jp
}

ecules, which is drastically different from the familiar swelling of isotropic gels mainly controlled by isotropic mixing interaction. ${ }^{8,9}$

The theoretical aspects of the swelling and phase behavior of LC gels in LC solvents have been investigated by many researchers, ${ }^{5-7,10,11}$ but the experimental survey still remains to be developed. Several researchers experimentally examined the deformation behavior of nematic networks swollen by nematic solvents under an electric field, ${ }^{12-15}$ but no detailed phase diagrams in each case were obtained. To the author's knowledge, there exists no quantitative experimental study for the swelling and phase behavior except for our previous papers. ${ }^{3,4}$ The present study focuses on how the amount of cross-linkage (cross-linking density) in the LC network influences the swelling and phase behavior. Crosslinking density $\left(C_{x}\right)$ is an important parameter of network structure, and it affects the elasticity and nematicity of the LC network each of which is directly related to one of the three governing forces (elastic force, nematic interaction, isotropic mixing interaction) for the equilibrium swelling: The network modulus is proportional to $C_{x}$ according to the principle of rubber elasticity; ${ }^{8,9}$ the nematic-isotropic transition temperature of dry LC network $\left(T_{\mathrm{NI}}^{N}\right)$ is considerably affected by $C_{x} \cdot{ }^{13,16-18}$ For example, the effect of $C_{x}$ on the volume transition temperature is an interesting issue. The volume transition takes place at the nematic-isotropic transition temperature $T_{\mathrm{NI}}^{G}$ for a swollen LC gel, namely, a mix- 
ture of nematic network and solvent. The $T_{\mathrm{NI}}^{G}$ strongly depends on the original nematicity of each component as well as the composition of the mixture in the equilibrium swollen state, i.e., the degree of equilibrium swelling. The nematicity of the network is closely related to $T_{\mathrm{NI}}^{N}$, while the swelling degree is significantly influenced by the network modulus as well as the nematicity of network. In addition to $T_{\mathrm{NI}}^{G}$, crosslinking density is expected to influence other aspects of volume transition phenomena such as the magnitude of discontinuous volume change at $T_{\mathrm{NI}}^{G}$.

In a previous paper, ${ }^{4}$ we showed that the swelling and phase behavior of the weakly cross-linked LC gels (with $C_{x}=1 \mathrm{~mol} \%$ ) were successfully described by the mean field theory. The data of the LC gels with widely different crosslinking densities (with $C_{x}=1-10 \mathrm{~mol} \%$ ) enable us to survey if the mean field theory correctly accounts for the effect of cross-linking density on volume transition. They also provide a detailed basis to discuss and develop the applicability of the LC gels as a temperature-sensitive soft actuator.

\section{THEORETICAL SCHEME}

In this section, we briefly summarize a mean field theory ${ }^{5-7}$ for a nematic network comprising rigid mesogen and flexible nonmesomorphic component (spacer) swollen in a nematic solvent. The equilibrium swelling and phase behavior is evaluated from a total free energy density $f$ composed of three terms,

$$
f=f_{m}+f_{e}+f_{n},
$$

where $f_{m}, f_{e}$, and $f_{n}$ are the free energy densities of isotropic mixing, network elasticity, and nematic ordering, respectively. The $f$ 's represent free energies per unit volume, namely, $f_{i}=F_{i} / N_{t}(i=m, e, n)$, where $F$ is the free energy. The total number of unit cells inside the gel $N_{t}$ is given by $N_{t}=n_{0} N_{0}+n N_{g}$, where $N_{0}$ and $N_{g}$ are the numbers of solvents inside the gel and the network chains, respectively, $n_{0}$ is the axial ratio of the nematic solvent (i.e., the number of sites occupied by one nematic solvent molecule), and $n$ is the total number of segments on a network chain. The axial ratios of rigid mesogen and non-mesomorphic component (spacer), designated as $n_{m}$ and $n_{s}$, respectively, are related to $n$ as $n=\left(n_{m}+n_{s}\right) / t$, where $t$ is the number of repeating units between adjacent cross-links.

On the basis of the Flory-Huggins theory, $f_{m}$ may be expressed as ${ }^{19}$

$$
\frac{f_{m}}{k T}=\frac{(1-\phi)}{n_{0}} \ln (1-\phi)+\chi \phi_{S}\left(1-\phi_{S}\right),
$$

where $k$ is the Boltzmann constant, $T$ is the absolute temperature. The volume fractions $\phi$ and $\phi_{s}$ are those of the network and spacer, respectively, and they are related as $\phi_{s}=\phi p$, where $p=n_{s} /\left(n_{m}+n_{s}\right)$ is the fraction of spacer segments. The degree of equilibrium swelling $Q$ is the inverse of $\phi$, namely, $\phi=Q^{-1}=V_{0} / V=n N_{g} / N_{t}$, where $V$ and $V_{0}$ are the volumes of the network in the fully swollen and dry states, respectively. The Flory-Huggins parameter $\chi$ characterizes the mixing interactions between nematic molecules and spacer segments. As shown in our previous study, ${ }^{4}$ the mix- ing interaction does not play an important role in the swelling of the nematic network in the nematic solvent. For simplicity, we assume here that the mesogen-spacer and nematogen-spacer mixing interactions are equivalent $\left(\chi_{m s}\right.$ $\left.=\chi_{0 s}\right)$; the nematogen acts as an athermal solvent for mesogen $\left(\chi_{m 0}=0\right)$.

Warner et al. $^{5,20}$ derived $f_{e}$ describing the energy change due to a deformation of nematic network upon swelling as a function of $\phi$ and orientational order parameter of mesogen $S_{m}$. It may be written as ${ }^{5,20}$

$$
\begin{aligned}
\frac{f_{e}}{k T}= & \frac{3}{2 n}\left\{\left[\frac{\phi}{\left(1+2 S_{m}\right)\left(1-S_{m}\right)^{2}}\right]^{1 / 3}\right. \\
& \left.+\frac{1}{3} \ln \left(1+2 S_{m}\right)\left(1-S_{m}\right)^{2}\right\} .
\end{aligned}
$$

In our previous study ${ }^{4}$ for weakly cross-linked nematic networks with high swellability $(\phi \ll 1)$, we employed another form $^{6,7}$ of $f_{e}$. This form ${ }^{6,7}$ is applicable to relatively dilute regime, and it becomes essentially the same as Eq. (3) in the dilute regime. ${ }^{21}$ In the present study, $\phi$ can range from dilute regime to moderately concentrated regime as cross-linking density is widely altered. We use here Eq. (3) as $f_{e}$ because it is applicable to the wide range of $\phi$.

The nematic free energy density $f_{n}$ may be given by the Maier-Saupe mean-field theory ${ }^{22,23}$ and its generalization to binary nematogens mixtures ${ }^{24,25}$ as follows:

$$
\begin{aligned}
\frac{f_{n}}{k T}= & \frac{\phi_{m}}{n_{m}} \int \psi\left(\theta_{m}\right) \ln 4 \pi \psi\left(\theta_{m}\right) d \Omega_{m} \\
& +\frac{\phi_{0}}{n_{0}} \int \psi\left(\theta_{0}\right) \ln 4 \pi \psi\left(\theta_{0}\right) d \Omega_{0}-\frac{1}{2} \nu_{m m} \phi_{m}^{2} S_{m}^{2} \\
& -\frac{1}{2} \nu_{00}(1-\phi)^{2} S_{0}^{2}-\nu_{m 0} \phi_{m}(1-\phi) S_{m} S_{0},
\end{aligned}
$$

where $d \Omega_{i}=2 \pi \sin \theta_{i} d \theta_{i}$, and $\theta_{i}(i=m, 0)$ is the angle between a reference axis and the director of each liquid crystal molecule. The subscripts $m$ and 0 refer to the mesogen and the solvent inside the gel, respectively, and $\phi_{m}$ is the volume fraction of the mesogen given by $\phi_{m}=\phi n_{m} /\left(n_{m}+n_{s}\right)$. The orientational order parameter $S_{i}(i=m, 0)$ is defined as

$$
S_{i}=\frac{1}{2}\left(3\left\langle\cos ^{2} \theta_{i}\right\rangle-1\right) .
$$

The angle bracket denotes the ensemble average defined as

$$
\left\langle\cos ^{2} \theta_{i}\right\rangle=\int \cos ^{2} \theta_{i} \psi\left(\theta_{i}\right) d\left(\cos \theta_{i}\right),
$$

where $\psi\left(\theta_{i}\right)(i=m, 0)$ is the normalized distribution function $\left(\int \psi\left(\theta_{i}\right) d \Omega_{i}=1\right)$ expressed by

$$
\psi\left(\theta_{i}\right)=\frac{1}{Z_{i}} \exp \left[\frac{1}{2} \eta_{i}\left(3 \cos ^{2} \theta_{i}-1\right)\right]
$$

using the constants $Z_{i}=\int \exp \left[\eta_{i}\left(3 \cos ^{2} \theta_{i}-1\right) / 2\right] d\left(\cos \theta_{i}\right)$ $(i=m, 0)$. The mean field parameters $\eta_{i}(i=m, 0)$ represent 
the strength of the nematic potentials and are respectively expressed in terms of $S_{m}$ and $S_{0}$ via minimization of the free energy with respect to $\psi\left(\theta_{i}\right)(i=m, 0)$,

$$
\begin{aligned}
\eta_{m}= & n_{m}\left[\nu_{m m} \phi_{m} S_{m}+\nu_{m 0}(1-\phi) S_{0}\right] \\
& -\frac{3 n_{m}}{n \phi_{m}\left(1+2 S_{m}\right)\left(1-S_{m}\right)^{2}} \\
& \times\left\{\left[\frac{\phi}{\left(1+2 S_{m}\right)\left(1-S_{m}\right)^{2}}\right]^{1 / 3}-1\right\} S_{m}\left(1-S_{m}\right)
\end{aligned}
$$

and

$$
\eta_{0}=n_{0}\left[\nu_{m 0} \phi_{m} S_{m}+\nu_{00}(1-\phi) S_{0}\right] .
$$

The Maier-Saupe nematic parameters $\nu_{m m}, \nu_{00}$, and $\nu_{m 0}$ characterize the mesogen-mesogen, the solvent-solvent, and the mesogen-solvent nematic interactions, respectively. They are inversely proportional to $T$, and $\nu_{00}$ is related to the nematic-isotropic transition temperature of the pure solvent $T_{\mathrm{NI}}^{S}$ at $\nu_{00}=4.54 T_{\mathrm{NI}}^{S} /\left(n_{0} T\right) .{ }^{23}$ The cross-interaction $\nu_{m 0}$ is not known, but assumed to be proportional to $\left(\nu_{m m} \nu_{00}\right)^{1 / 2}$ with a proportionality constant $\kappa$ as $\nu_{m 0}=\kappa\left(\nu_{m m} \nu_{00}\right)^{1 / 2} \cdot 11,25$ The constant $\kappa$ characterizes the relative strength of the cross-interaction as compared to that in the same nematogens. The condition $\kappa=1$ corresponds to an ideal case where all nematic molecules interact with equal strength.

The chemical potentials of the solvents inside and outside the gel $\left(\mu_{0}\right.$ and $\mu_{0}^{o}$, respectively), given by $\mu=\phi$ $-\phi(\partial f / \partial \phi)$, are balanced in swelling equilibrium,

$$
\mu_{0}\left(\phi, S_{m}, S_{0}\right)=\mu_{0}^{o}\left(S_{b}\right)
$$

where the order parameter of the pure solvent outside the gel $S_{b}$ is given by Eq. (5) with

$$
\eta_{b}=n_{0} \nu_{00} S_{b} .
$$

Thus the equilibrium conditions is given by the following equation:

$$
\begin{aligned}
& \frac{1}{n}\left\{\left[\frac{\phi}{\left(1+2 S_{m}\right)\left(1-S_{m}\right)^{2}}\right]^{1 / 3}+\frac{1}{2} \ln \left(1+2 S_{m}\right)\left(1-S_{m}\right)^{2}\right\} \\
& \quad+\frac{1}{n_{0}} \ln (1-\phi)+\frac{\phi}{n_{0}}+\chi \phi_{s}^{2}+\frac{1}{2} \nu_{m m} S_{m}^{2} \phi_{m}^{2} \\
& \quad+\nu_{m 0} S_{m} \phi_{m}(1-\phi)+\frac{1}{2} \nu_{00}\left[S_{0}^{2}(1-\phi)^{2}-S_{b}^{2}\right] \\
& \quad+\frac{1}{n_{0}} \ln \frac{Z_{b}}{Z_{0}}=0 .
\end{aligned}
$$

The equilibrium values for $Q(=1 / \phi), S_{m}, S_{0}$, and $S_{b}$ as a function of $T$ can be obtained by solving Eq. (5) ( $i$ $=m, 0, b)$ and Eq. (12) self-consistently.

\section{EXPERIMENT}

The side-chain LC networks LCN-I or LCN-II were prepared by radical copolymerization of the mesogenic acrylate monomer I or II (Fig. 1) and 1,6-hexanediol diacrylate (cross-linker), respectively. The monomers I and II were syn-

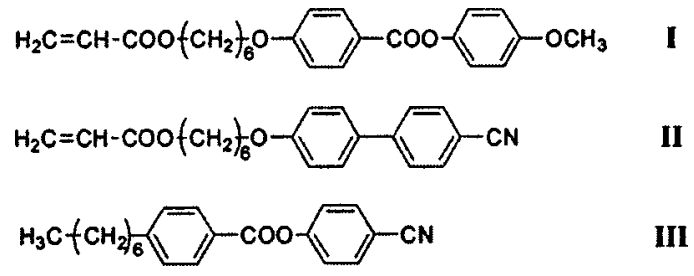

FIG. 1. Molecular structures of the employed liquid crystalline monomers and solvent.

thesized after the methods in Refs. 26 and 27. The concentration of the initiator $2,2^{\prime}$-azobis(isobutyronitrile) was 1 mol \% in the total reactants. The amounts of cross-linker in the total reactants introduced during synthesis were varied from 1 to $10 \mathrm{~mol} \%$, as shown in Table I. We call this parameter the cross-linking density $\left(C_{x}\right.$ mol $\left.\%\right)$ although the true cross-linking density is presumably smaller than the cross-linker concentration during synthesis because the cross-linking efficiency is reduced by the presence of the mesogen.

The reactant mixtures dissolved in toluene were polymerized in capillaries with inner diameter of several hundreds microns at $80^{\circ} \mathrm{C}$, where the LC monomers were in the isotropic phase. After the polymerization, the cylindrical gels were extracted from the capillaries, washed with toluene to remove unreacted molecules. The gels were then gradually deswollen in successive mixtures of toluene and methanol, increasing the methanol content in each step. The fully deswollen gels were completely dried in air. The nematicisotropic transition temperatures of the dry (pure) LC networks $\left(T_{\mathrm{NI}}^{N}\right)$, estimated in the cooling process by polarizing optical microscopy, are tabulated in Table I. The dry gels were allowed to swell in the low molar mass liquid crystal III (Fig. 1). The LC solvent III with a quoted purity of at least 99\% was used without further purification.

A hot stage (Linkam LK-600PM) polarizing microscope (Nikon E600 POL) with a camera was used for the measurement of gel diameters and the phase observation. The details of the measurements were described elsewhere. ${ }^{3,4,28}$ The surface level of the LC solvent in an optical cell was adjusted to be low enough so that the LC phases inside the gels as well as the boundary of the gel surface were distinctly visible when viewed through the microscope (but high enough to

TABLE I. Cross-linking density, nematic-isotropic transition temperatures of the LC networks in the dry and fully swollen states and volume fractions of network in the vicinity of volume transition temperatures.

\begin{tabular}{lcccc}
\hline \hline Network sample & $C_{x}$ & $T_{\mathrm{NI}}^{N \mathrm{a} /{ }^{\circ} \mathrm{C}}$ & $T_{\mathrm{NI}}^{G} /{ }^{\circ} \mathrm{C}$ & $\phi_{\mathrm{NI}}^{\mathrm{b}}$ \\
\hline LCN-I-1 & 1 & 125 & 58.2 & 0.074 \\
LCNI-1.5 & 1.5 & 123 & 59.3 & 0.123 \\
LCN-I-3 & 3 & 121 & 59.7 & 0.181 \\
LCN-I-10 & 10 & $107 \pm 3^{\mathrm{c}}$ & 62.0 & 0.376 \\
LCN-II-1 & 1 & 131 & 71.0 & 0.183 \\
LCN-II-3 & 3 & 126 & 73.0 & 0.333 \\
LCN-II-10 & 10 & $120 \pm 2^{\mathrm{c}}$ & $73.2 \pm 0.5^{\mathrm{c}}$ & 0.412 \\
\hline
\end{tabular}

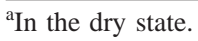

${ }^{\text {b } V o l u m e ~ f r a c t i o n ~ o f ~ n e t w o r k ~ i n ~ t h e ~ s w o l l e n ~ p h a s e ~ a t ~} T=T_{\mathrm{NI}}^{G}+0.2{ }^{\circ} \mathrm{C}$.

${ }^{\mathrm{c}}$ Broad transition. 
immerse the gel completely). The gels swelled isotropically even in the nematic phase due to the polydomain LC structure where a high nematic order exists locally but the orientation of directors is globally random in space. The swelling of the gel was equilibrated at each temperature, and the gel diameter was measured on a enlarged photograph as a function of temperature. The degree of equilibrium swelling $(Q)$ was determined from the ratio of the gel diameters in the dry and swollen states $\left(d_{0}\right.$ and $d_{s}$, respectively): $Q=V / V_{0}$ $=\left(d_{S} / d_{0}\right)^{3}$, where $V$ and $V_{0}$ are the gel volumes in the swollen and dry states, respectively.

\section{RESULTS AND DISCUSSION}

Part (a) of Figs. 2 and 3 shows the temperature $(T)$ dependence of the degree of equilibrium swelling $(Q)$ for LCN-I and LCN-II with different cross-linking densities $\left(C_{x}\right)$, respectively. In the figures, absolute temperature is reduced by the nematic-isotropic transition temperature of the pure LC solvent III $\left(T_{\mathrm{NI}}^{S}=327.2 \mathrm{~K}\right)$. The data were obtained in the cooling process. Our previous studies ${ }^{3,4}$ revealed no significant difference in the swelling and phase behavior between the cooling and heating processes for LCN-I-1 and LCN-II-1 in some nematic solvents. All the systems have the two independent nematic-isotropic transition temperatures $T_{\mathrm{NI}}^{G}$ and $T_{\mathrm{NI}}^{S}: T_{\mathrm{NI}}^{G}$ (indicated by arrows in the figures) is that for the LC molecules inside the gel, while $T_{\mathrm{NI}}^{S}$ is for the LC solvent outside the gel. In the range $T<T_{\mathrm{NI}}^{G}$, the nematic network and the miscible nematic solvent inside the gel form a single nematic phase. Phase separation inside the equilibrium swollen gel was not observed by polarizing microscopy with a typical magnification used in the studies ${ }^{29,30}$ on phase separation of LC mixtures.

There exists three characteristic temperature regions depending on the LC phases inside and outside the gel: totally isotropic phase $\left(T>T_{\mathrm{NI}}^{G}\right)$; totally nematic phase $\left(T<T_{\mathrm{NI}}^{S}\right)$; nematic phase inside the gel and isotropic phase outside the gel $\left(T_{\mathrm{NI}}^{S}<T<T_{\mathrm{NI}}^{G}\right)$. It is clearly seen that except for LCNI-10 and LCN-II-10 with the highest $C_{x}$, the swelling behavior strongly correlates with the phase characteristics. Qualitatively, the correlation is independent of $C_{x}$ as well as the chemical structure of mesogen. In the totally isotropic phase $T>T_{\mathrm{NI}}^{G}, Q$ slightly decreases with decreasing $T$. At $T$ $=T_{\mathrm{NI}}^{G}$, the swollen isotropic gel discontinuously shrinks into the nematic gel, i.e., volume transition takes place as a result of nematic ordering inside the gel. In the range $T_{\mathrm{NI}}^{S}<T$ $<T_{\mathrm{NI}}^{G}$, where the LC phases inside and outside are nematic and isotropic, respectively, the nematic gel reswells with descending $T$, and the magnitude of $Q$ at $T \approx T_{\mathrm{NI}}^{S}$ becomes comparable to those in the region $T>T_{\mathrm{NI}}^{G}$. The nematic ordering outside the gel at $T_{\mathrm{NI}}^{S}$ yields a kink in the swelling curve without discontinuity. In the totally nematic phase $T<T_{\mathrm{NI}}^{S}$, $Q$ is almost independent of $T$. Essentially the same $T$ dependence of $Q$ was observed in LCN-II-1 swollen in a nematogen similar to the mesogen II, ${ }^{4}$ which suggests that this characteristic swelling behavior is not due to the dissimilarity between mesogen and nematogen; it is universal for the nematic network in a miscible nematic solvent.

As is evident from Figs. 2(a) and 3(a), an increase in
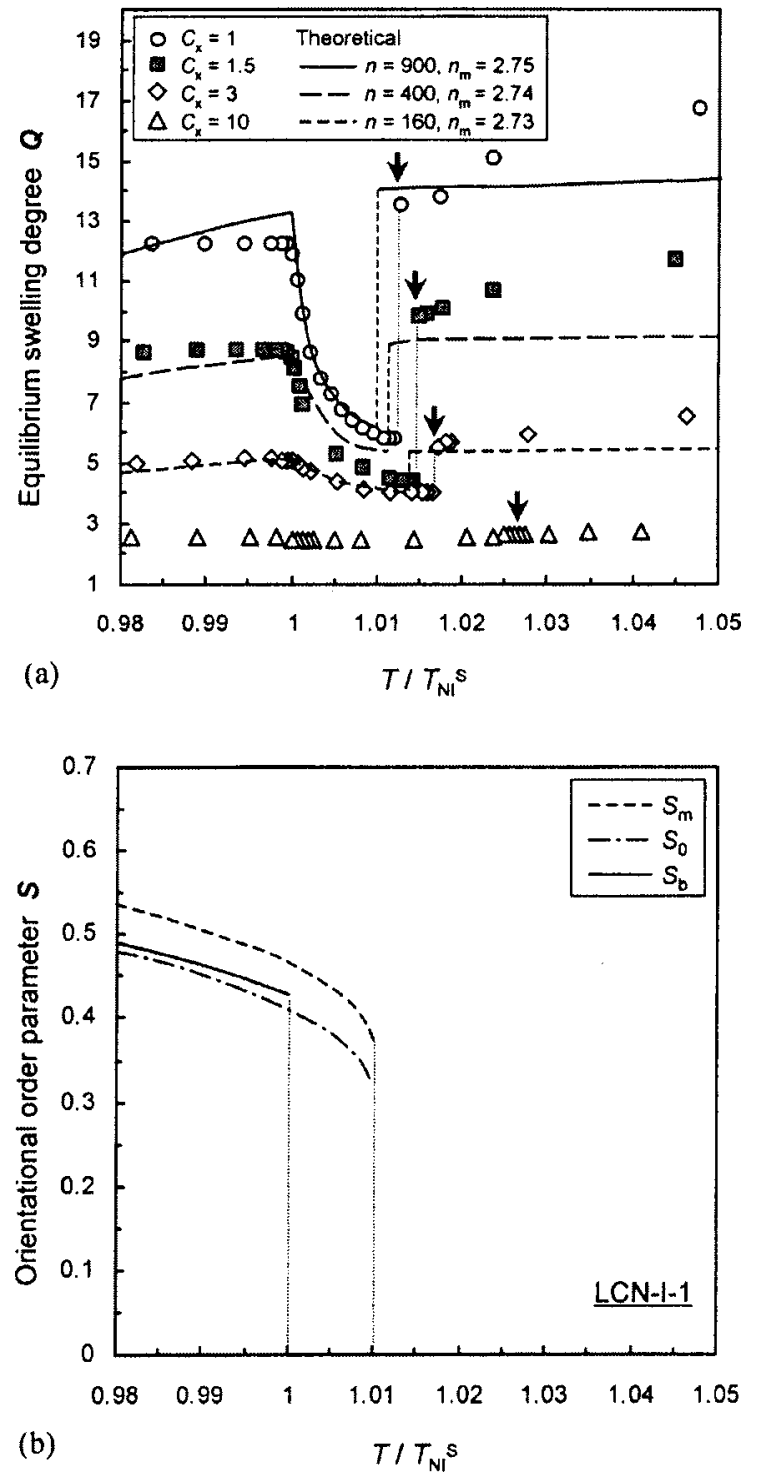

FIG. 2. (a) Equilibrium swelling degree $(Q)$ of LCN-I with different crosslinking densities $\left(C_{x}\right)$ as a function of absolute temperature $(T)$ reduced by the nematic-isotropic transition temperature of the pure nematic solvent $T_{\mathrm{NI}}^{S}=327.2 \mathrm{~K}$. The arrows indicate $T_{\mathrm{NI}}^{G}$ for each gel. The lines represent the fitted theoretical swelling curves with $n=900$ and $n_{m}=2.75$ for LCN-I-1, $n=400$ and $n_{m}=2.74$ for LCN-I-1.5, $n=160$ and $n_{m}=2.73$ for LCN-I-3. The values of the other parameters used are $n_{0}=2.5, p=0.14, \nu_{00} / \chi$ $=0.3, \nu_{m m} / \nu_{00}=1.24, \kappa=0.9007$. (b) The orientational order parameters of the mesogen on the network $\left(S_{m}\right)$, solvent inside the gel $\left(S_{0}\right)$, solvent outside the gel $\left(S_{b}\right)$ as a function of $T / T_{\mathrm{NI}}^{S}$ calculated using the mean field theory with the same parameter values as (a) for LCN-I-1.

cross-linking density yields the following two significant effects on the swelling and phase behavior: (i) decrease in the magnitude of discontinuous volume change at $T_{\mathrm{NI}}^{G}$; (ii) shift of $T_{\mathrm{NI}}^{G}$ to higher temperatures. These effects were commonly observed for both LCN-I and LCN-II. The former is the direct consequence of an increase in network modulus due to an increment in $C_{x}$. As the network modulus increases, the network becomes more resistant to the expansion, which lessens the swellability of network. The decrease in swellability depresses the difference in $Q$ between the fully swollen and shrunken states. The result of LCN-I-10 $\left(C_{x}\right.$ $=10 \mathrm{~mol} \%$ ) indicates the characteristics of an extreme case 

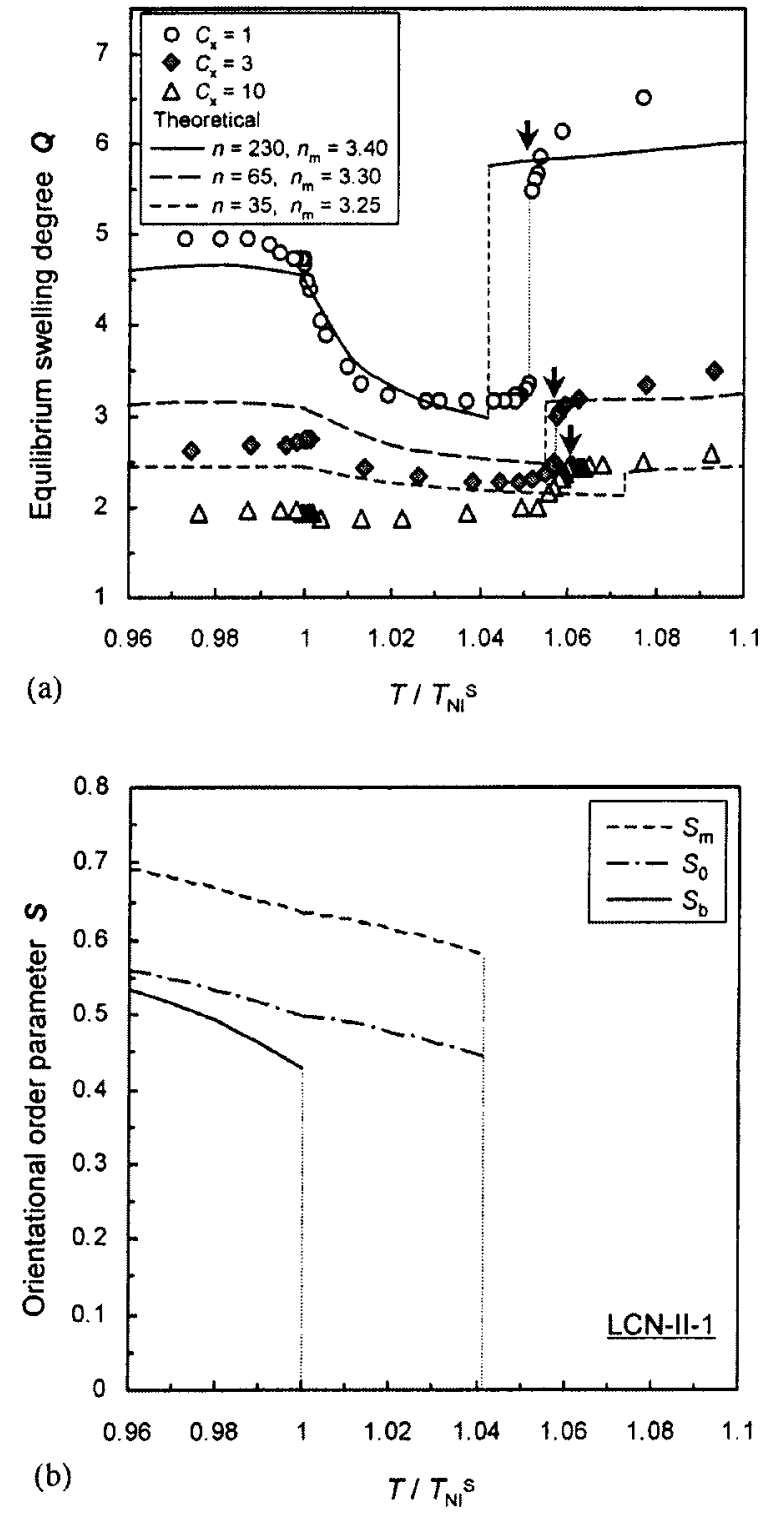

FIG. 3. (a) Equilibrium swelling degree $(Q)$ of LCN-II with different crosslinking densities $\left(C_{x}\right)$ as a function of absolute temperature $(T)$ reduced by the nematic-isotropic transition temperature of the pure nematic solvent $T_{\mathrm{NI}}^{S}=327.2 \mathrm{~K}$. The arrows indicate $T_{\mathrm{NI}}^{G}$ for each gel. The lines represent the fitted theoretical swelling curves with $n=230$ and $n_{m}=3.40$ for LCN-II-1, $n=65$ and $n_{m}=3.30$ for LCN-II-3, $n=35$ and $n_{m}=3.25$ for LCN-II-10. The values of the other parameters used are $n_{0}=2.5, p=0.13, \nu_{00} / \chi=0.2$, $\nu_{m m} / \nu_{00}=1.032, \kappa=0.9991$. (b) The orientational order parameters of the mesogen on network $\left(S_{m}\right)$, solvent inside the gel $\left(S_{0}\right)$, solvent outside the gel $\left(S_{b}\right)$ as a function of $T / T_{\mathrm{NI}}^{S}$ calculated using the mean field theory with the same parameter values as (a) for LCN-II-1.

of high cross-linking density: The network modulus is so high that the system exhibits no appreciable change of $Q$ in response to the nematic ordering inside and outside the gel. For LCN-II-10 with the similarly high cross-linking density, a slight and continuous volume change was observed at around $T_{\mathrm{NI}}^{G}$ accompanying the broad nematic-isotropic transition over the temperature range of $\sim 1{ }^{\circ} \mathrm{C}$. This broad transition in the swollen gel stems from the rather broad transition of the LC network backbone (in the dry state) over the range of $\sim 4{ }^{\circ} \mathrm{C}$. The broadening of the phase transition was also reported for some dry LC networks with high cross-

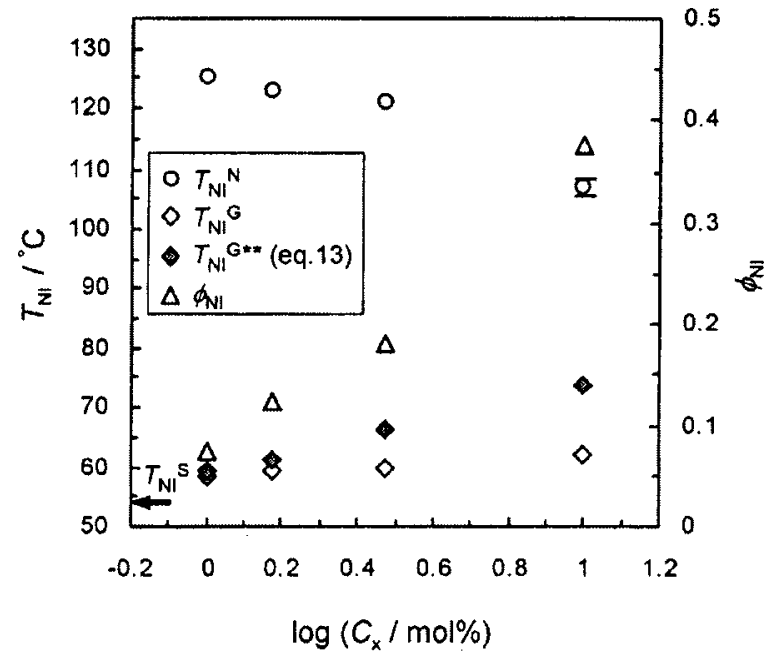

FIG. 4. The nematic-isotropic transition temperatures of LCN-I in the dry and fully swollen states ( $T_{\mathrm{NI}}^{N}$ and $T_{\mathrm{NI}}^{G}$, respectively), volume fractions of network $\left(\phi_{\mathrm{NI}}\right)$ in the vicinity of $T_{\mathrm{NI}}^{G}\left(T=T_{\mathrm{NI}}^{G}+0.2^{\circ} \mathrm{C}\right)$, and $T_{\mathrm{NI}}^{G * *}$ calculated by Eq. (13) as a function of cross-linking density $\left(C_{x}\right)$. The arrow indicates the nematic-isotropic transition temperature of the pure LC solvent III $\left(T_{\mathrm{NI}}^{S}=54.2^{\circ} \mathrm{C}\right)$.

linking densities. ${ }^{18}$ It may originate from the heterogeneous distribution of cross-links acting as defects for nematic phase formation.

As can be seen in Table I, the latter effect (ii) is opposite to the effect of $C_{x}$ on $T_{\mathrm{NI}}^{N}$ (the nematic-isotropic transition temperature of the dry LC networks): An increase in $C_{x}$ yields a shift of $T_{\mathrm{NI}}^{G}$ to higher temperatures, while it reduces $T_{\mathrm{NI}}^{N}$. Figures 4 and 5 display $T_{\mathrm{NI}}^{G}$ and $T_{\mathrm{NI}}^{N}$ of LCN-I and $\mathrm{LCN}-\mathrm{II}$ as a function of $C_{x}$, respectively. The trend for $T_{\mathrm{NI}}^{N}$ observed qualitatively accords with the results in the earlier studies for some dry LC networks with different crosslinking densities prepared in the isotropic phase..$^{13,16-18}$ To understand the dependence of $T_{\mathrm{NI}}^{G}$ on $C_{x}$, it should be no-

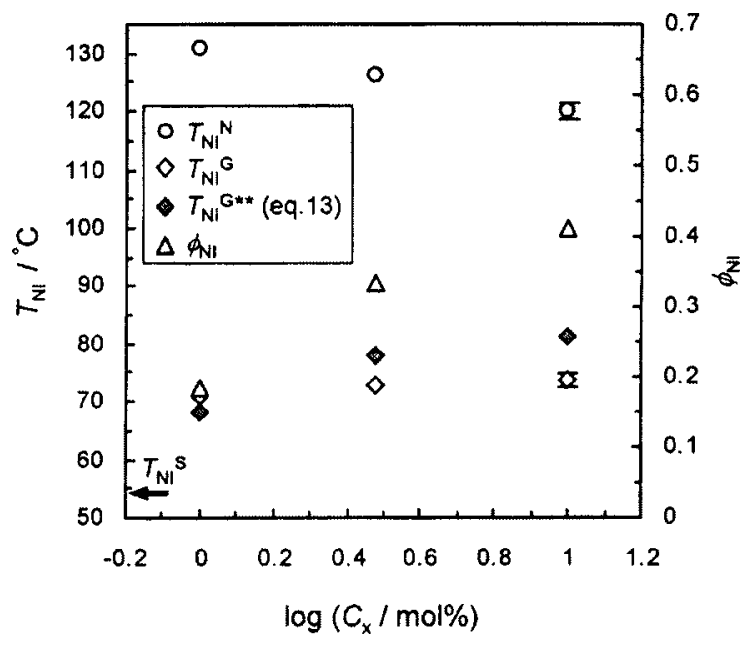

FIG. 5. The nematic-isotropic transition temperatures of LCN-II in the dry and fully swollen states $\left(T_{\mathrm{NI}}^{N}\right.$ and $T_{\mathrm{NI}}^{G}$, respectively), volume fractions of network $\left(\phi_{\mathrm{NI}}\right)$ in the vicinity of $T_{\mathrm{NI}}^{G}\left(T=T_{\mathrm{NI}}^{G}+0.2^{\circ} \mathrm{C}\right)$, and $T_{\mathrm{NI}}^{G * *}$ calculated by Eq. (13) as a function of cross-linking density $\left(C_{x}\right)$. The arrow indicates the nematic-isotropic transition temperature of the pure LC solvent III $\left(T_{\mathrm{NI}}^{S}=54.2^{\circ} \mathrm{C}\right)$. 
ticed that $T_{\mathrm{NI}}^{G}$ for a swollen gel reflects the characteristics of a mixture of the LC network and the LC solvent; the LC network content in the swollen gel increases with increasing $C_{x}$; an increment in $C_{x}$ reduces $T_{\mathrm{NI}}^{N}$, but still $T_{\mathrm{NI}}^{N}$ $\left(>100{ }^{\circ} \mathrm{C}\right)$ is much higher than $T_{\mathrm{NI}}^{S}\left(=54.2^{\circ} \mathrm{C}\right)$. The transition (absolute) temperature for a LC mixture defined by the following simple additivity $\left(T_{\mathrm{NI}}^{G * *}\right)$ is helpful for the qualitative understanding along this consideration,

$$
T_{\mathrm{NI}}^{G * *}=\left(1-\phi_{\mathrm{NI}}\right) T_{\mathrm{NI}}^{S}+\phi_{\mathrm{NI}} T_{\mathrm{NI}}^{N}
$$

where $\phi_{\mathrm{NI}}$ is the volume fraction of network in the vicinity of $T_{\mathrm{NI}}^{G}$ (at $T=T_{\mathrm{NI}}^{G}+0.2{ }^{\circ} \mathrm{C}$ ) in the swollen phase. The calculated value of $T_{\mathrm{NI}}^{G *}$ as well as $\phi_{\mathrm{NI}}$ for each network are also shown in Figs. 4 and 5. The approach based on Eq. (13) is rather primitive, but qualitatively shows that $T_{\mathrm{NI}}^{G * *}$ increases with $C_{x}$ as a result of an increase in the LC network content $\left(\phi_{\mathrm{NI}}\right)$ with higher transition temperature $\left(T_{\mathrm{NI}}^{N}>T_{\mathrm{NI}}^{S}\right)$. The values of $T_{\mathrm{NI}}^{G * *}$ are in good agreement with $T_{\mathrm{NI}}^{G}$ for the LCN-I and LCN-II with the low cross-linking densities $C_{x}$ $<1.5 \mathrm{~mol} \%$ (i.e., low $\phi_{\mathrm{NI}}$ ). However, the $T_{\mathrm{NI}}^{G * *}$ values are fairly higher than $T_{\mathrm{NI}}^{G}$ for the strongly-cross-linked networks with $C_{x}>3 \mathrm{~mol} \%$ (i.e., high $\phi_{\mathrm{NI}}$ ), and the deviation becomes larger with increasing $C_{x}$ (or $\phi_{\mathrm{NI}}$ ). This tendency is apparently similar to the dependence of $T_{\mathrm{NI}}$ for miscible mixtures of low molar mass LCs on the blend composition: ${ }^{25,31}$ In the phase diagram for the miscible LC mixtures without strong cross-interaction, the composition$T_{\mathrm{NI}}$ curve becomes convex downward; the simple additivity for transition temperatures is rather successful only in the low concentration limit of each component, while the estimates from the additivity are fairly higher than the real transition temperatures at intermediate concentrations. ${ }^{25,31}$

To account for the experimental results by the mean field theory, the theoretical predictions are compared to the experimental data in Figs. 2 and 3. The lines in Figs. 2(a) and 3(a) represent the fitted theoretical swelling curves. In the data-fit, LCN-I-10 without appreciable volume change against $T$ was excluded because the theoretical applicability of Gaussian chain to the stiff network is very doubtful. Figures 2(b) and 3(b) illustrate the orientation order parameter of each LC, i.e., mesogen on network $\left(S_{m}\right)$, LC solvent molecules inside and outside gel ( $S_{0}$ and $S_{b}$, respectively) which was simultaneously obtained in the data-fit for LCN-I and LCN-II-1, respectively. The quantities $n, n_{0}, n_{m}, p$, $\nu_{00} / \chi, \nu_{m m} / \nu_{00}, \kappa$ were employed as fitting parameters. As first demonstrated for the weakly cross-linked LC networks with $C_{x}=1 \mathrm{~mol} \%,{ }^{4}$ the mean field theory successfully reproduces the complicated swelling and phase characteristics observed, and in addition, the theory demonstrates that the nematic order mainly governs the swelling behavior: The formation of a single nematic phase of mesogen and nematogen inside the gel at $T_{\mathrm{NI}}^{G}$ (i.e., simultaneous jumps of $S_{m}$ and $S_{0}$ from zero to finite values) drives a discontinuous volume decrease; in the range $T_{\mathrm{NI}}^{S}<T<T_{\mathrm{NI}}^{G}$, where there exists no nematic order outside the gel $\left(S_{b}=0\right)$, an increase in nematic order inside the gel $\left(S_{m}\right.$ and $\left.S_{0}\right)$ upon cooling thermodynamically induces the further uptake of solvent by the network (reswelling); $Q$ is almost independent of $T$ in the totally isotropic phase $T>T_{\mathrm{NI}}^{G}$ without nematic order $\left(S_{m}\right.$ $=S_{0}=S_{b}=0$ ) as well as in the totally nematic phase $T$ $<T_{\mathrm{NI}}^{S}$ with comparable nematic orders inside and outside the gel $\left(S_{m} \approx S_{0} \approx S_{b}\right)$. It should be noted that the successful data-fit is substantially attributed to the framework of the mean field theory, instead of many fitting parameters. In fact, the parameters affect only the magnitude of $Q$ and the location of $T_{\mathrm{NI}}^{G}$.

In the data-fit for LCN-I or LCN-II with different $C_{x}$, the two parameters $n$ (the number of segment units between neighboring cross-links) and $n_{m}$ (axial ratio of mesogen) were altered, while the values of other parameters were fixed: $n_{0}=2.5, p=0.14, \quad \nu_{00} / \chi=0.3, \quad \nu_{m m} / \nu_{00}=1.24, \kappa$ $=0.9007$ for LCN-I, and $n_{0}=2.5, p=0.13, \nu_{00} / \chi=0.2$, $\nu_{m m} / \nu_{00}=1.032, \kappa=0.9991$ for LCN-II were consistently used in the data-fitting. The parameter $n$ is directly related to cross-linking density, and $n_{m}$ governs the nematicity of pure LC network. As described before, it was experimentally observed that an increase in $C_{x}$ reduced $T_{\mathrm{NI}}^{N}$, i.e., nematicity of the pure LC network. The theoretical value of $T_{\mathrm{NI}}^{N}$ is defined as the temperature at which $S_{m}$ jumps from zero to a finite value when $\phi=1$. The numerical calculations show that $T_{\mathrm{NI}}^{N}$ is not appreciably influenced by $n$. This implies that the cross-linking density effect on $T_{\mathrm{NI}}^{N}$ observed mainly results from the "chemical effect." The cross-link acts as defects for nematic phase formation. The quantity $n_{m}$ is originally defined as the axial ratio of mesogen. Meanwhile, in the framework of the mean field theory, the absolute value of $n_{m}$ is also requested to reflect the effects concerning the nematicity, i.e., the above-mentioned chemical effect and the interaction between network backbone and dangling mesogen none of which are explicitly considered in the theory. ${ }^{5-7,10-12}$ As the total effect related to nematicity already appears in $T_{\mathrm{NI}}^{N}$ observed, $n_{m}$ can reflect the effects via the adjustment of the theoretical $T_{\mathrm{NI}}^{N}$ to the experimental value by altering $n_{m} \cdot{ }^{25,32}$ The nematic parameter $\nu_{m m}$ also affects $T_{\mathrm{NI}}^{N}$, but the influence was too small to reproduce the difference in $T_{\mathrm{NI}}^{N}$ observed. The fitted values of $n_{m}$ were determined so that the theoretical predictions could reproduce the most successfully the experimental results for both $T_{\mathrm{NI}}^{N}$ and the $Q-T$ curve: $n_{m}=2.75,2.74,2.73$ for LCN-I-1, $-1.5,-3$, and $n_{m}$ $=3.40,3.30,3.25$ for LCN-II-1, $-3,-10$, respectively. The resulting theoretical values of $T_{\mathrm{NI}}^{N} / T_{\mathrm{NI}}^{S}$ for LCN-I-1, -1.5 , -3 are $1.22,1.22,1.21$, while the corresponding experimental values are 1.22, 1.21, 1.20, respectively; for LCN-II-1, -3 , and -10 , the theoretical values are $1.27,1.23,1.21$, while the concerning experimental values are 1.24, 1.22, 1.20 , respectively.

As can be seen in Figs. 2(a) and 3(a), the theoretical prediction successfully describes the characteristics of the cross-linking density effects: an increase in $C_{x}$ (i.e., a decrease in $n$ ) yields an reduction in network swellability, a shift of $T_{\mathrm{NI}}^{G}$ to higher temperatures, and a decrease in the magnitude of discontinuous volume change at $T_{\mathrm{NI}}^{G}$. It should be noticed that the theory is successful in reproducing simultaneously the positive dependence of $T_{\mathrm{NI}}^{G}$ and the negative dependence of $T_{\mathrm{NI}}^{N}$ on $C_{x}$. This suggests that elastic modulus of network significantly affects $T_{\mathrm{NI}}^{G}$. High network modulus 
lessens the swellability of the network, which increases the LC network content (whose $T_{\mathrm{NI}}^{N}$ is much higher than $T_{\mathrm{NI}}^{S}$ of the solvent) in the swollen LC gel. This effect is much stronger than the depressing effect of cross-linking density on the nematicity of the pure LC network.

Cross-linking density may influence the isotropic mixing interaction $(\chi)$ and/or the nematic interactions $\left(\nu_{m m}, \kappa\right)$. In fact, the quality of the data-fit will be further improved if we use other parameters such as $\chi, \nu_{m m}, \kappa$ as additional adjustable parameters. In the data-fit, however, we altered here the minimum numbers of the parameters $\left(n\right.$ and $\left.n_{m}\right)$ in order to demonstrate unambiguously that the framework of the mean field theory is substantially successful in describing the effect of cross-linking density on the swelling and phase behavior. The fitted values of $n$ do not coincide with the real segment numbers between adjacent cross-links, but it does not revoke the validity of the theory in view of the roughness included in the mean field approach based on lattice model as well as the ambiguity in $C_{x}$ without taking the effective crosslinkage into account.

\section{CONCLUSIONS}

The effect of cross-linking density $\left(C_{x}\right)$ on nematic ordering-induced volume transition of LC networks in a miscible nematic solvent has been studied. An increase in $C_{x}$ reduces swellability and nematic stability (nematic-isotropic transition temperature $T_{\mathrm{NI}}^{N}$ ) of the pure (dry) LC networks. Nematic ordering inside the swollen LC gel at $T_{\mathrm{NI}}^{G}$ induces a discontinuous change in gel volume. An increment in $C_{x}$ yields a depression in the magnitude of the discontinuous volume change as well as a shift of $T_{\mathrm{NI}}^{G}$ to higher temperatures. The latter effect, which is opposite to the influence of $C_{x}$ on $T_{\mathrm{NI}}^{N}$, is qualitatively because the fraction of network (whose $T_{\mathrm{NI}}^{N}$ is much higher than the nematic-isotropic transition temperature of the solvent $T_{\mathrm{NI}}^{S}$ ) in the gel increases due to a decrease in swellability as $C_{x}$ increases. No appreciable volume transition is observed for the strongly-cross-linked LC networks with $C_{x}=10 \mathrm{~mol} \%$ in the nematic solvent, although they exhibit the nematic-isotropic transition. The mean field theory for nematic gel satisfactorily describes the effects of cross-linking density on the swelling and phase behavior observed.

\section{ACKNOWLEDGMENT}

The numerical calculation was performed with the approval of Academic Center for Computing and Media Studies, Kyoto University.

${ }^{1}$ Responsive Gels: Volume Transition I, edited by K. Dusek, Adv. Polym. Sci. (Springer-Verlag, Berlin and Heidelberg, 1993), Vol. 109.

${ }^{2}$ Responsive Gels: Volume Transition II, edited by K. Dusek, Adv. Polym. Sci. (Springer-Verlag, Berlin and Heidelberg, 1993), Vol. 110.

${ }^{3}$ K. Urayama, Y. Okuno, T. Kawamura, and S. Kohjiya, Macromolecules 35, 4567 (2002).

${ }^{4}$ K. Urayama, Y. Okuno, T. Nakao, and S. Kohjiya, J. Chem. Phys. 118, 2903 (2003)

${ }^{5}$ X. J. Wang and M. Warner, Macromol. Theory Simul. 6, 37 (1997).

${ }^{6}$ A. Matsuyama and T. Kato, Phys. Rev. E 64, 010701 (2001).

${ }^{7}$ A. Matsuyama and T. Kato, J. Chem. Phys. 116, 8175 (2002).

${ }^{8}$ T. R. G. Treloar, Physics of Rubber Elasticity, 3rd ed. (Oxford University Press, Oxford, 1975).

${ }^{9}$ B. Erman and J. E. Mark, Structure and Properties of Rubberlike Networks (Oxford University Press, Oxford, 1997).

${ }^{10}$ F. Brochard, J. Phys. (Paris) 40, 1049 (1979)

${ }^{11}$ F. Benmouna, U. Maschke, X. Coqueret, and M. Benmouna, Polym. Int. 50, 469 (2001).

${ }^{12}$ R. Zentel, Liq. Cryst. 1, 589 (1986).

${ }^{13}$ N. R. Barnes, F. J. Davis, and G. R. Mitchell, Mol. Cryst. Liq. Cryst. 168, 13 (1989).

${ }^{14}$ R. Kishi, Y. Suzuki, H. Ichijo, and O. Hirasa, Chem. Lett. 1994, 2257.

${ }^{15}$ R. Kishi, Y. Suzuki, H. Ichijo, and O. Hirasa, Mol. Cryst. Liq. Cryst. 295, 113 (1997).

${ }^{16}$ R. Zentel and G. Reckert, Makromol. Chem. 187, 1915 (1986).

${ }^{17}$ G. R. Mitchell, F. J. Davis, and A. Ashman, Polymer 28, 639 (1987).

${ }^{18}$ Y. Zhao and G. Yuan, Macromolecules 29, 1067 (1996).

${ }^{19}$ P. J. Flory, Principles of Polymer Chemistry (Cornell University, Ithaca, 1953).

${ }^{20}$ M. Warner, K. P. Gelling, and T. A. Vilgis, J. Chem. Phys. 88, 4008 (1988).

${ }^{21}$ A. Matsuyama and T. Kato, J. Chem. Phys. 114, 3817 (2001).

${ }^{22}$ W. Maier and A. Z. Saupe, Z. Naturforsch. A 14a, 882 (1959).

${ }^{23}$ P.-G. de Gennes, The Physics of Liquid Crystals, 2nd ed. (Oxford, New York, 1993)

${ }^{24}$ F. Brochard, J. Jouffroy, and P. Levinson, J. Phys. (Paris) 45, 1125 (1984).

${ }^{25}$ H.-W. Chiu and T. Kyu, J. Chem. Phys. 103, 7471 (1995).

${ }^{26}$ M. Portugall, H. Ringsdorf, and R. Zentel, Makromol. Chem. 183, 2311 (1982).

${ }^{27}$ V. P. Shibaev, S. G. Kostromin, and N. A. Plate, Eur. Polym. J. 18, 651 (1982).

${ }^{28}$ M. De Sarkar, K. Urayama, T. Kawamura, and S. Kohjiya, Liq. Cryst. 27, 795 (2000).

${ }^{29}$ F. Benmouna, J. Ruhe, and D. Johansmann, Liq. Cryst. 26, 1655 (1999).

${ }^{30}$ D. Nwabunma and T. Kyu, Macromolecules 32, 664 (1999).

${ }^{31}$ E. C.-H. Hsu and J. F. Johnson, Mol. Cryst. Liq. Cryst. 20, 177 (1973).

${ }^{32}$ H.-W. Chiu, Z. L. Zhou, T. Kyu, G. L. Cada, and L. C. Chien, Macromolecules 29, 1051 (1996). 